Prévenir et guérir à l'âge de la nature corrompue : Le Pourtraict de la santé (1606) de Joseph du Chesne

\title{
Violaine Giacomotto-Charra
}

\section{(2) OpenEdition}

Édition électronique

URL : http://journals.openedition.org/edl/906

DOI : $10.4000 /$ edl. 906

ISSN : 2296-5084

Éditeur

Université de Lausanne

\section{Édition imprimée}

Date de publication : 15 décembre 2015

ISBN : 978-2-940331-47-5

ISSN : 0014-2026

\section{Référence électronique}

Violaine Giacomotto-Charra, « Prévenir et guérir à l'âge de la nature corrompue : Le Pourtraict de la santé (1606) de Joseph du Chesne », Études de lettres [En ligne], 3-4 | 2015, mis en ligne le 01 décembre 2018, consulté le 17 décembre 2020. URL : http://journals.openedition.org/edl/906 ; DOI : https://doi.org/10.4000/edl.906 


\section{PRÉVENIR ET GUÉRIR À LÂGE DE LA NATURE CORROMPUE: LE POURTRAICT DE LA SANTÉ (1606) DE JOSEPH DU CHESNE}

Dans la pensée savante de la fin de la Renaissance, la notion de corruption est d'abord une notion philosophique aristotélicienne majeure, inséparable de son corollaire, la génération. Le traité De la génération et de la corruption est en effet, depuis le XIII ${ }^{e}$ siècle, au cœur de l'enseignement de la philosophie de la nature tel qu'il est dispensé dans les universités. Selon cette première acception, le terme corruptio désigne, dans la théorie de la forme et de la matière, le mouvement par lequel se perd la forme substantielle d'un être, prélude nécessaire à une nouvelle naissance. Dans un sens différent, c'est également une notion importante héritée de la tradition médicale antique, dans laquelle la notion d'airs corrompus, par exemple, joue un rôle crucial, en particulier pour toute la médecine qui se réclame de l'héritage hippocratique. Enfin, le terme de corruption peut avoir un sens plus banal, tant au sens physique (pourrissement, putréfaction, tuméfaction) que moral. Cette toute dernière valeur n'est pas anodine en une période troublée par les angoisses eschatologiques et les lendemains difficiles des conflits religieux. Cette communication se propose d'explorer les différents sens du mot et leur évolution, dans un texte qui se trouve lui-même à la croisée des chemins, puisqu'il s'agit du régime de santé du médecin paracelsiste Joseph Duchesne qui écrit son Pourtraict de la santé pour apprendre au lecteur à préserver la santé de son corps, aussi bien que celle de son âme.

La corruption est d'abord, au moment où écrit Joseph du Chesne (15461609), une notion pleinement aristotélicienne, inséparable de son corollaire, la génération: les libri naturales du Stagirite demeurent le socle de l'apprentissage de la philosophie de la nature dans les universités, ainsi que celui de toute réflexion ultérieure sur cette dernière. La 
publication de commentaires se poursuit ${ }^{1}$, et l'essentiel de la philosophie aristotélicienne, dont une connaissance moins technique était depuis longtemps diffusée en français par des voies aussi diverses que la poésie d'idées ou le dialogue philosophique, est désormais disponible de manière plus systématique en langue vulgaire avec La Physique (1603) de Scipion Dupleix ${ }^{2}$. Le terme corruptio désigne ainsi, suivant la théorie de la forme et de la matière, le mouvement par lequel se perd la forme substantielle d'un être. La corruption, cependant, n'est pas uniquement une mort, elle est le prélude nécessaire à une nouvelle naissance, fût-ce, comme le rappelle Dupleix, la mort d'un homme et la naissance d'une charogne ${ }^{3}$ :

Le cours ordinaire generalement estably en toute la nature des choses est tel qu'il est impossible qu'une chose se corrompe et meure qu'une autre ne s'engendre et renaisse: ny au contraire qu'une chose s'engendre et naisse qu'une autre ne se corrompe et meure ${ }^{4}$.

Génération et corruption ne consistent donc pas simplement en l'union et la désunion des corps, mais en celles de l'être dans son entier. La corruption ne doit en particulier pas être confondue avec l'altération, car contrairement à cette dernière, qui est un simple changement qualitatif, la corruption ne s'inscrit pas dans la durée:

Si la generation et corruption se faisoient avec quelque espace de temps, une chose recevroit son estre par pieces, c'est-à-dire, seroit en partie et en partie ne seroit pas 5 .

I. Voir G. Zabarella, De Rebus Naturalibus libri XXX, ou les Commentarii Collegii Conimbricensis S. I. in duos libros de generatione et corruptione Aristotelis Stagirite.

2. La Physique ou sciences des choses naturelles parut pour la première fois à Paris en 1603. Signe de son succès considérable et du poids des théories qu'elle véhicule, elle fut rééditée une vingtaine de fois entre cette date et 1645 , avec des corrections et des additions pour plusieurs éditions. Nous suivons l'édition de Roger Ariew, fac simile de celle de 1640, à Rouen, chez Louis du Mesnil.

3. "Quand un enfant naist, nous ne daignons pas dire que la naissance apporte la corruption de la semence dont il est engendré, et quand l'homme meurt nous ne daignons pas dire que sa corruption est suivie de la generation d'une charongne, parce que telles choses sont viles en la bouche des hommes" (S. Dupleix, La Physique ou sciences des choses naturelles, p. 220).

4. Ibid., p. 219.

5. S. Dupleix, La Curiosité naturelle, f. $121 \mathrm{v}^{\circ}-122 \mathrm{r}^{\circ}$. 
Ainsi donc, "la generation et la corruption regardent tout l'estre de la chose, l'une pour l'establir, l'autre pour le destruire» ${ }^{6}$.

C'est dans cet univers intellectuel qui n'ignore rien du sens premier de ces notions que s'inscrit Le Pourtraict de la santé (1606) du médecin paracelsiste Joseph du Chesne ${ }^{7}$. Si celles-ci, cependant, demeurent formellement liées à l'aristotélisme en raison de l'importance capitale du De generatione et corruptione, elles se sont compliquées, modifiées, nuancées au cours des siècles, au fil des commentaires scolastiques d'abord, puis dans le cadre d'un syncrétisme renaissant particulièrement puissant. La philosophie naturelle de Du Chesne lui-même est encore mal connue, mais, comme l'a récemment montré Hiro Hiraï, à la fois étroitement dépendante de la philosophie aristotélicienne et de la médecine hippocratique, en même temps que fortement marquée par le néoplatonisme et les influences de la pensée hermétique et "chymique», en particulier le modèle de l'Idea medicina philosophica de Pierre Séverin ${ }^{8}$. Or, parce que le Pourtraict de la santé est un traité de diététique dans la lignée des «régimes de santé» qui fleurissent depuis le Moyen Âge, il ne saurait abstraire les soins du corps de la considération générale de l'univers: il permet de mieux comprendre comment s'articulent, aux divers niveaux de la Création, la notion de "corruption" avec ses développements particuliers, liés à l'exercice effectif de la médecine, ainsi que d'en percevoir les déplacements, les transformations et les enjeux.

$\mathrm{Du}$ Chesne, en effet, prend soin de dessiner les cadres conceptuels dans lesquels s'inscrit son traité grâce à sa dédicace à Henry de Bourbon,

6. S. Dupleix, La Physique ou sciences des choses naturelles, p. 221.

7. J. du Chesne, Le Pourtraict de la santé. Médecin de Henri IV, Du Chesne est surtout connu pour son rôle dans la diffusion des idées de Paracelse, car il est apparu au début du XVII e siècle comme le porte-parole des «médecins chymistes». En réalité, "son paracelsime était bien modéré» (H. Hiraï, "Paracelsisme, néoplatonisme et médecine hermétique dans la théorie de la matière de Joseph Du Chesne à travers son Ad veritatem hermetica medicine (1604)»). Il faut également signaler la présence du paracelsime dans son œuvre la plus connue, La Morocosmie: ou De la folie, vanité, et inconstance du Monde. Bien qu'il introduise dans son Pourtraict certaines notions "chymiques», le socle hippocrato-galénique de la médecine élémentaire et humorale y demeure très présent et ce n'est pas à la dimension paracelsienne de ses préconisations diététiques que nous nous intéressons ici.

8. Voir H. Hiraï, «Paracelsisme, néoplatonisme et médecine hermétique dans la théorie de la matière de Joseph Du Chesne à travers son Ad veritatem hermetica medicine (1604)». 
puis à un chapitre liminaire capital, le «Proème». Si la dédicace, d'abord, est le lieu d'une apologie attendue de la diététique, elle replace également la théorisation de cette discipline ancienne dans la perspective fondamentale des liens entre l'homme et l'univers, qui ne consistent pas uniquement en l'analogie du microcosme et du macrocosme:

Entre tel nombre de belles qualitez, et opinions, il me semble que celle qui convient le mieux [à la nature humaine], et qui approche le plus de la verité, c'est quand on luy attribue le nom d'harmonie, d'accord, et de proportion: nom, dont l'ont baptisée la pluspart [des anciens philosophes] fort à propos. Car par là, c'est faire apparoir en l'homme, non la seule harmonie des cieux en ses mouvemens si reglez, et exactement mesurez, selon le dire des Pythagoriciens: non le grand rapport et symbole seulement, des choses superieures, avec les inferieures [...] selon les Platoniciens: ains c'est recognoistre particulierement encore en l'homme, une admirable liaison, tres-estroitte conjonction, et harmonie en toutes ses parties: non seulement pour le regard de l'ame, mais aussi du corps, qui ne pourroit aucunement subsister, sans ceste belle harmonie, symmetrie et proportion naturelle, qui reside aux humeurs elementaires, dont il est composé 9

$\mathrm{Du}$ Chesne peut ainsi introduire par une longue comparaison entre le corps humain et l'instrument de musique, dont il convient de savoir conserver, dans les deux cas, "l'accord et harmonie», sa justification et sa définition de la diététique qui perpétuent la tradition hippocratique d'une médecine préventive plutôt que curative. L'harmonie évoquée par la dédicace, cependant, n'est que théorique; parce que cette dernière consiste, selon les codes du genre, en un éloge topique, elle ne fonde la diététique qu'elle suppose que dans un univers parfait mais caduque, dont l'histoire chrétienne a modifié le cours. C'est en réalité le "Proème» qui livre au lecteur la clef de l'ouvrage:

Car c'est chose certaine que Dieu qui est la mesme bonté et perfection, crea dès le commencement toutes choses bonnes et en leurs especes parfaictes pour l'usage et benefice de l'homme [...] afin de luy servir comme d'un sacrement et certaine asseurance que tant qu'il persevereroit à luy obeyr et complaire, sa vie seroit immortelle. Mais depuis, sa revolte estant intervenuë, il s'est trouvé decheu avec toute sa posterité

9. J. Du Chesne, Le Pourtraict de la santé, "Dedicace à Henry de Bourbon [...]", [n. p.]. 
de ceste grace et felicité, et tombé en si grands malheurs, qu'il a esté comme degradé et despoüillé de tous les dons excellens et de l'esprit et du corps dont auparavant il avoit esté revestu ${ }^{10}$.

S'il n'est pas original, surtout en milieu protestant, de souligner les conséquences physico-biologiques de la Chute, celles-ci prennent pour le médecin un relief particulier, car le dérèglement de l'ordre originel, punition de la transgression, modifie le statut du corps:

Son corps qui auparavant jouyssoit d'une si bonne symmetrie et temperature, est maintenant tant et tant intemperé que l'homme se peut dire le plus miserable d'entre tous les animaux: d'autant qu'il est affligé luy seul de plus de diverses et grandes maladies, que tous les autres ne peuvent estre: estant ordinairement comme un vray esgoust de tous maux ${ }^{11}$.

Du Chesne prend donc soin de décrire également ce phénomène en termes philosophiques, pour inscrire la question de la diététique dans une logique ontologique ainsi que, christianisme oblige, eschatologique:

Aussi certes par ceste desobeissance, et la terre en a esté maudite, et maudits de mesme par consequent les autres elemens: si bien qu'en lieu d'entretenir la vie de l'homme incorruptible, ils ont participé euxmesmes d'une estrange alteration et corruption, de laquelle il faut un jour qu'ils soient restaurez ${ }^{12}$.

Or, il se produit ici un double déplacement: le médecin fait basculer du côté de la corruption la notion, toute différente, d'altération; en sens inverse, la corruption cesse d'être un passage pour devenir un état. Un tel glissement peut être le signe d'une difficulté théologique: fautil considérer les transformations de la nature et de l'homme après la Chute comme une altération ou comme une véritable corruption, c'està-dire un changement d'être? L'homme rendu corruptible par la volonté de Dieu est-il devenu un homme intrinsèquement corrompu, au sens où l'homme du XVII e siècle peut être considéré comme le fruit de la corruption d'Adam? Si Du Chesne s'est ici posé ces questions, il ne se

\footnotetext{
Io. Ibid., p. 5.

II. Ibid., p. 7.

I2. Ibid., p. 7 sq.
} 
hasarde pas, cependant, sur les chemins de la théologie, mais considère plutôt les deux notions sous leur aspect pratique. Devenue synonyme de "dégradation", en effet, l'altération annonce ou dévoile la corruption à l'œuvre: elle se fait symptôme. Celle-ci, par ailleurs, ne marque pas seulement les corps, elle est, par la faute de l'homme, empreinte dans la nature même:

La terre auparavant beniste, et en toutes sortes plantureuse, fut maudite et rendue sterile: et au lieu que tous les fruicts creez pour l'usage de l'homme estoient fruicts delicieux et utiles, et autant de secrettes teinctures et quintes essences balsamiques, qui le preserveroient de toute corruption: ce sont maintenant au contraire fruicts pernicieux, fruicts grossiers, fruicts terrestres, pleins de tartre, d'ordure, de lies et de tant d'impuretez et corruptions ${ }^{13}$.

L'inscription dans la réalité tangible de l'idée chrétienne, et non plus aristotélicienne, de corruption se traduit ainsi par une forme de perversion de l'ordre élémentaire du monde: ce monde, que l'on peut qualifier lui-même de corrompu, est en même temps le facteur et le vecteur de la corruption, dont les manifestations sensibles prennent la forme de la pourriture, de la souillure et de l'ordure. On pourrait arguer que Du Chesne utilise ici des sens différents du mot, si le glissement du sens philosophique au sens courant de "putréfaction" n'était explicitement présenté comme un lien de cause à effet: l'existence d'une corruption visible et permanente n'est rien d'autre que la manifestation sensible d'une nature rendue intrinsèquement corruptible. Cette dernière a donc pris pour le médecin le hideux visage que dessinent, depuis l'automne de la Renaissance, la montée en puissance des inquiétudes eschatologiques et l'abondante littérature apocalyptique, qui ne décrit pas uniquement la fin du monde mais s'attarde sur sa déchéance et sur son vieillissement, renouvelant en une version sombre la vieille analogie du microcosme et du macrocosme ${ }^{14}$. Le "Proème» de Du Chesne esquisse ainsi une semblable nature, qui ne paraît plus rien conserver de la vénusté de celle de la Renaissance.

I3. Ibid., p. 8.

I4. Un bon exemple s'en trouve dans M. Quillian, La Derniere Semaine ou consommation du monde. Voir T. Victoria, Un Livre de feu dans un siècle de fer. 
Que la nature du médecin corresponde bien à la vision noire qu'en ont la plupart de ses contemporains n'est pas surprenant, mais pas non plus sans conséquences, car cela modifie de facto le rôle de la diététique. Du Chesne, on l'aura noté, souligne que la nature originellement créée par Dieu produisait "secrettes teinctures et quintes essences balsamiques" afin de préserver le corps de "toute corruption»: le médecin, et particulièrement le médecin "chymiste", est donc celui qui va pouvoir, par la pratique de sa discipline, tenter d'atténuer les conséquences physiologiques de la chute. Le discours théorique de la «Dédicace», qui faisait du corps un instrument harmonieux et de la diététique l'art de le maintenir accordé, est transformé par le «Proème», d'où se dégage l'idée d'une diététique comme palliatif des conséquences physico-biologiques du péché, remède physique d'une déchéance plus profonde. Le Pourtraict de la santé n'est pas simplement un régime de santé pour mieux vivre ou bien vivre, mais un «régime de vivre» ${ }^{15}$.

Cette perspective conduit $\mathrm{Du}$ Chesne à réaffirmer, tout en les renouvelant, les éléments de la tradition, en particulier la dépendance du corps aux mouvements de la sphère céleste d'une part, aux six choses non naturelles de l'autre ${ }^{16}$. L'idée que le déséquilibre ou la maladie sont les fruits de la corruption devenue inhérente au fonctionnement intrinsèque de la nature fortifie ainsi chez lui la certitude que le médecin doit être également philosophe. L'idée n'est pas neuve, mais il y insiste tout particulièrement:

Les grands et vrais medecins doivent avoir la cognoissance de l'univers: cognoistre tous les astres du Ciel, avoir la notice de leurs mouvemens divers, et de leurs influences, qui font les saisons differentes et mutations de temps, d'où naissent plusieurs dissemblables changemens en nos corps, et s'engendrent en eux diverses maladies. Ils doivent aussi cognoistre les differences des vapeurs froides et humides, dont se font les nuages, pluyes, neiges, frimats et semblables: et des exhalaisons chaudes et seiches, comme sont les esclairs, tonnerres, foudres, et tels autres meteores souffreux, et bruslans [...]. Tel vray medecin doit de

I5. J. Du Chesne, Le Pourtraict de la santé, p. 9.

I6. Il s'agit dans la tradition médicale des éléments considérés comme extérieurs au corps mais influant sur son devenir, soit dans l'ordre canonique: air, boire et manger, inanition et réplétion, veille et sommeil, travail et repos, perturbations de l'âme. 
mesme avoir cognoissance de tout ce qui est produict et contenu en l'Element de l'eau, [etc.] ${ }^{17}$.

L'exercice de la médecine doit donc commencer par l'exploration attentive de tous les phénomènes impliquant les mouvements particuliers du monde sublunaire, mais surtout de ceux dans lesquels les transformations qualitatives quotidiennes prennent un caractère spectaculaire et témoignent de manière visible de l'inconstance et de la violence des phénomènes, signes du dérèglement de la nature originelle. La tradition hippocratique de l'observation locale (Du Chesne mentionne le traité De l'air, des lieux et des eaux, dont il revendique l'autorité $^{18}$ ) est de la même manière renouvelée par l'idée que la puissance de la nature s'exprime également désormais dans l'apparition de maladies nouvelles. Lorsque Du Chesne insiste sur le rôle formateur des voyages, ce n'est pas tant, comme il le dit, pour "savoir les maladies qui pullulent d'ordinaire le plus en tel lieu» ${ }^{19}$, que pour faire la preuve, comme le montrent les exemples qu'il choisit, quapparaissent sans cesse de nouveaux maux. Il conclut ainsi de la description d'une maladie apparue en Angleterre en 1486:

Voilà le seul remede, qu'on a trouvé à un tel mal, mal et remede, qui n’ont esté descrits, ny par Hippocrates, ny par Galien: mais le medecin qui aura voyagé en Angleterre, le pourra avoir appris [...] et non par la lecture des livres des anciens Grecs, Latins, ny Arabes ${ }^{20}$.

Il s'agit donc bien de légitimer une évolution du paradigme médical, qui suppose à la fois de retrouver ces remèdes balsamiques chers aux médecins "chymiques» et dont le péché a effacé la trace, et la traque de la corruption à l'œuvre à travers ses manifestations tangibles partout où elles se trouvent.

Un point de l'œuvre particulièrement frappant est en effet la manière dont $\mathrm{Du}$ Chesne s'applique à décrire très minutieusement les deux ensembles de phénomènes dont le «Proème» signale l'importance: les

I7. J. Du Chesne, Le Pourtraict de la santé, "Quel est l'office de tout vray medecin", p. 147 sq.

I8. Ibid., p. 150.

19. Ibid.

2o. Ibid., p. 152. 
effets sublunaires du mouvement des astres d'une part, les choses non naturelles de l'autre. Il traite ensemble le premier thème et la description des mouvements de l'air, dans un chapitre consacré à ce dernier. L'air, en effet, est d'abord pour le médecin l'élément essentiel à la vie en même temps que le vecteur traditionnel des contagions; il est ensuite, au sens cosmologique du terme, le lieu des météores où s'observe le mieux le jeu des influences célestes; et c'est enfin un élément qui revêt, dans le système philosophique de Du Chesne, une valeur particulière ${ }^{21}$. Il constitue donc, dans l'élaboration d'une diététique adaptée au monde corrompu, un élément capital. Or il devient, sous la plume du médecin, tant en raison de sa pensée propre que d'une longue tradition météorologique et médicale qui s'intéresse à "la sphère de l'air troublé» ${ }^{22}$ et aux airs corrompus, une sorte de clef pour la compréhension des choses. Il est en quelque sorte le double symbole de la "malédiction" de la terre consécutive à la chute et de la manière dont le corps, désormais corruptible, est contraint de subir les influences délétères du monde qui, paradoxalement, le nourrit en même temps qu'il le détruit:

Mais d'autant que c'est l'air qu'on inspire, qui principalement s'infecte de telles malignes vapeurs, et que ledit air pour le symbole qu'il y a de chose spirituelle à chose spirituelle, et de vapeur à vapeur nous communique ceste infection par l'inspiration ordinaire que nous tirons de luy: il nous faut parler principalement de ceste chose non naturelle, tant et tant importante ${ }^{23}$.

Du Chesne souligne ainsi deux de ses aspects fondamentaux, qui sont comme l'illustration de la théorie développée par le "Proème»: l'influence des mouvements des astres et la production des météores donnent corps au principe généralisé d'altération ${ }^{24}$; le thème des airs pestilents, à celui de corruption.

L'air est en effet, dans la pensée de Du Chesne, un élément de nature spirituelle, ce qui le rend particulièrement inapte à résister aux

2I. Sur ce point, voir H. Hiraï, "Paracelsisme, néoplatonisme et médecine hermétique dans la théorie de la matière de Joseph Du Chesne à travers son Adveritatem hermetice medicine (1604)", p. 27 sq.

22. Ch. Deluz, «La sphère de l'air troublé».

23. J. Du Chesne, Le Pourtraict de la santé, p. 158.

24. Sur le sujet des météores, voir en particulier le chapitre consacré au vent, ibid., p. $169-182$. 
changements d'état ${ }^{25}$, et le texte n'a de cesse de recenser les "diverses mutations de l'air", "changemens et mutations en l'air» ou "si subites et grandes mutations à l'air» qui sont l'ordinaire de la vie quotidienne ${ }^{26}$. Mais si la tradition ancienne des correspondances entre les humeurs, les différents cycles temporels et le mouvement des astres est si longuement évoquée, c'est afin de souligner la quasi-infinité des variations ainsi produites, causes de l'altération des corps et donc signe de corruption:

Voilà les grandes et diverses mutations des qualitez de l'air aux quatre saisons de l'an, aux mois, aux jours et aux nuicts: choses dignes d'estre remarquées de tous medecins, d'autant qu'elles apportent diverses alterations aux corps, et aux humeurs des sains et des malades. [...] Ce qui importe grandement d'estre sçeu du medecin, veu que non seulement les corps des sains, ains que ceux des malades encore d'avantage reçoivent grande alteration selon les divers mouvemens de l'air ${ }^{27}$.

L'air, par ailleurs, n’est pas seulement, «Prothée» ou «Cameleon», le parangon du muable, il est aussi et surtout le lieu et le vecteur de la corruption. C'est ici l'ampleur du lexique déployé par Du Chesne pour rendre compte de ce phénomène qui trahit son extrême intérêt:

L'air auparavant tousjours serain, et qui estoit comme la mesme pureté pour purifier les esprits de l'homme, desquels il estoit le soustien, l'entretien et la principale nourriture, est au contraire depuis devenu trouble, nebuleux et le plus souvent infect et pestilent ${ }^{28}$. L'air trouble, nuageux, pluvieux, aquatique, grossier, corrompu et infect, rendu tel

25. "Or l'air comme c'est une vapeur subtile et spirituelle, c'est pourquoy il est subjet à si divers changemens, et propres à recevoir si diverses alterations [...]: c'est aussi pourquoy nostre temperament en est si souvent changé et perverty» (ibid., p. 159). Il y a sans doute quelque chose de l'ordre d'une fascination pour le mouvement et la transformation, comme en témoignent les images utilisées par Du Chesne, par exemple dans le passage suivant: "tant y a que comme un Prothée, ou comme un Cameleon est subject à changer de diverses faces et couleurs" (ibid., p. 178). On peut également signaler l'incontestable souffle épique de certains passages consacrés aux météores: "de ce resserrement, survient un grand combat, esmotion, et agitation, entre le chaud souphreux et l'aëreux humide: qui estant contrainct, par son plus fort ennemy, de s'enfuyr, s'eslargit, fend ce qui l'environne, fait ouverture à travers l'air, et se fait vent, soufflant, sifflant, ronflant, bruyant, tempestant, bourdonnant» (ibid., p. 170).

26. Voir ibid., l'ensemble du chapitre «De l'air».

27. Ibid., p. 161.

28. Ibid., p. 8. 
par les lacs, estangs, marescages, eaux dormantes et corrompües de cloaques, gouffres, et centres de la terre: bestes mortes, ou telles autres infections et puanteurs, proches des habitations, est un air nuisible, pernicieux, pestilent et contraire à la conservation de nostre vie. Et au contraire l'air plus serain et exempt de toutes ces mauvaises qualitez, est celuy qu'on doit choisir pour sa demeure, à fin de vivre sainement et longuement ${ }^{29}$.

Les deux champs lexicaux ainsi mis en parallèle ("trouble», "nebuleux», "infect", "pestilent», "grossier», "corrompu », "nuisible», "pernicieux", etc., versus "serain») signalent non seulement, s'il était besoin, les enjeux liés à la corruption, mais aussi, nettement, que "corruption» n'est plus ici uniquement le corollaire de "génération" mais également et surtout le contraire de "pureté», sans pour autant abdiquer le sens aristotélicien. De manière significative, les termes qui servent à décrire ce symbole de la corruption de nature que sont les airs pestilents, sont ceux-là mêmes qui traduisaient, dans le «Proème», l'état de l'homme déchu:

[...] le beau rayon de son intelligence [...] a esté depuis ombragé et obscurcy de tant d'espais broüillars et nuages», «ses beaux sens ont esté tellement depravez [...] qu'ils l'abbaissent maintenant jusques aux plus profondes abysmes de la terre ${ }^{30}$.

Le corps, "esgoust de tous maux", matérialise par ses incessantes sécrétions la corruption qui le ronge:

[...] ses yeux clair-voyans sont maintenant pleins de sussusions, mailles et cataractes: son nez doux-fleurant est maintenant une sentine de puanteur et corruption ${ }^{31}$.

De ce fait, le régime de santé proposé par Du Chesne, qui s'appuie en bonne part, il convient de le rappeler, sur la diététique de ses prédécesseurs, apparaît néanmoins plus spécifiquement comme une pratique de purification et de désinfection. Il faut souligner l'importance qu'il accorde à l'hygiène: outre qu'il convient de localiser sa maison dans un lieu aéré (car les vents, mus par la «divine Providence [...] sont les

29. Ibid., p. 178.

3o. Ibid., p. 4 sq.

3I. Ibid., p. 6. 
nettoyeurs et purificateurs des immondices et superfluitez " ${ }^{22}$ ), il faut aussi veiller à ce que celle-ci soit «tousjours bien nette, [...] que nulle ordure ny puanteur soit de fumiers d'estable, soit de la cuisine, ne la puisse tant soit peu infecter». Il faut également veiller à conserver les fenêtres closes si d'aventure l'air était «nuageux, trouble, pruineux ou pluvieux" ${ }^{33}$. Ces préconisations ne sont pas uniquement individuelles et l'on voit s'esquisser une hygiène urbaine relativement nouvelle, s'appuyant sur l'exemple de Toulouse, qui ayant élargi ses rues et les faisant nettoyer quotidiennement, n'a plus jamais été «subjecte à contagion " ${ }^{34}$ :

L'air infect par les charongnes mortes, par les boües et ordures puantes, qui sont ou parmy les ruës, ou pres du lieu de l'habitation, se peut changer et corriger, en les ostant et en bien nettoyant lesdits lieux sales: à quoy une bonne police est requise ${ }^{35}$.

Pour les soins du corps, Du Chesne met également l'accent sur la propreté: aller à la garde-robe "tout aussi tost qu'[on] sera levé» et user de divers remèdes si l'on ne parvient pas à évacuer les excréments (obsession bien connue de la purge et du lavement), se frotter le visage, se "curer les oreilles", «se nettoyer et bien frotter les dents, avec la racine preparée de guimauves» ${ }^{36}$, humer certaine herbe qui "aydera à destouper le nez, et à bien faire moucher " ${ }^{37}$, bref, vider ces "sentines de corruption" que sont devenus, comme le rappelait le "Proème», nos organes sensoriels. La même inquiétude gouverne certains aspects des préceptes spécifiquement diététiques: il faut se méfier des concombres, car «ils se corrompent facilement et engendrent des fievres putrides " ${ }^{38}$, des fraises, car «elles se corrompent facilement dans l'estomach, d'où peuvent s'engendrer des fievres" ${ }^{39}$. Le vinaigre, en revanche, "empesche la corruption [...] et d'avantage il est fort necessaire en temps de peste ${ }^{40}$, et c'est en bon Gascon que Du Chesne recommande la consommation de

32. Ibid., p. 175.

33. Ibid., p. 358.

34. Voir J. Du Chesne, Le Pourtraict de la santé, p. 179 sq.

35. Ibid., p. 179.

36. Ibid., p. 361.

37. Ibid., p. 363.

38. Ibid., p. 390.

39. Ibid., p. 363.

40. Ibid., p. 487. 
l'ail: "c'est en outre la theriaque des vilageois en Gascongne contre les pestes et le mauvais air: les enfans qui en usent ne sont jamais sujects aux corruptions et vermines " ${ }^{41}$. Maladie et saleté sont ainsi confondues en une même souillure, et l'on notera tout particulièrement que, déjà, l'animal sauvage est considéré comme meilleur que l'animal domestique, "car la nourriture qui en survient est moins excrementeuse que les autres: d'autant que telles bestes sauvages vivent en un air plus libre, eslargy et plus sec. [...] Aussi on voit d'ordinaire comme les chairs de telles sauvagines se conservent sans se corrompre, beaucoup plus long temps que les domestiques" ${ }^{42}$. On notera enfin que, même dans le domaine diététique, la dimension morale attachée à la notion de corruption n'est jamais bien loin, puisque, aussi bonne que soient les viandes sauvages, on évitera de les consommer «quand elles sont en ruth et en amour» ${ }^{43}$.

Les préceptes diététiques sur lesquels débouchent in fine le Pourtraict de la santé témoignent donc, malgré l'aspect banal de certaines recommandations, de l'importance de la notion de corruption dans la représentation que se fait Du Chesne du monde qui l'entoure: tout élément sujet à corruption, au sens de "pourriture", participe du grand dessein de corruption qui frappe l'homme depuis la Chute. Cette corruption ne vaut pas seulement comme signe: conformément au mouvement qui la lie à la génération, la corruption engendre, mais elle ne donne plus naissance qu'à des choses néfastes, en particulier les fièvres, ellesmêmes qualifiées de "putrides". La superposition analogique des acceptions philosophiques, médicales et ordinaires est ainsi particulièrement exemplaire dans le cas de l'huile:

Car l'huile resiste mieux que le beurre, aux putrefactions: Le beurre se corrompt bien-tost s'il n'est fondu ou salé: Et l'huile se conserve longuement de soy-mesme sans souffrir alteration: Voire c'est un Antidote et preservatif, contre les plus grands venins et corruptions, et si est l'ennemy capital de toute vermine ${ }^{44}$.

4I. Ibid., p. 380.

42. Ibid., p. 413.

43. Ibid., p. 413 sq.

44. Ibid., p. 485 sq. 
Le danger de corruption, qui guette tous les niveaux de l'être, légitime ainsi l'idéal d'une vie saine mais également morale. On peut en effet noter que se dessine clairement, chez Du Chesne, une hygiène sociale qui fait de l'ordure la marque de certaines catégories de population. Il préconise ainsi de situer sa maison "[si c'est en ville] en ruë spacieuse, esloignée d'artisans et du menu peuple " ${ }^{45}$, de même qu'il reconnaît s'adresser "particulierement aux riches, non aux pauvres et mechaniques, ausquels tels regimes ne sont propres». L'hygiène des femmes, pour qui «la netteté et proprieté est tousjours plus requise que la saleté et l'ordure», est également un point sur lequel il insiste, mais ici, le conseil hygiénique a pour but de conformer l'apparence féminine à un critère de pureté qui est aussi un canon esthétique, comme "avoir leurs mains blanches et doüilletes» ou "conserver les dents sans se gaster et corrompre» ${ }^{46}$. Enfin se développe un idéal de vie rustique, éloigné des affaires et de la cour, le modèle à fuir étant celui des courtisans, dont la vie est décrite dans les mêmes termes que les météores: "en continuelle action trouble et perturbation", ils sont "oragez par les aspres tourbillons de l'ambition et de l'envie ${ }^{47}$.

Cette dernière remarque nous permet d'aborder un ultime aspect de la prégnance de la notion de corruption dans la pensée de Du Chesne: son lien avec les perturbations de l'âme, "qui ne sont autre chose qu'un estrange et subit mouvement d'un esprit boüillant, turbulent et esgaré " ${ }^{48}$. La corruption quotidienne n'est pas seulement signe du destin de l'Adam déchu, elle est aussi manifestation tangible d'ignominies plus secrètes, dont la révélation brutale peut ainsi permettre la guérison par l'horreur qu'elles provoquent. Le traitement que préconise en dernier recours Du Chesne pour remédier aux atteintes «de l'amour voluptueux", passion de l'âme à laquelle il consacre le plus long des chapitres concernés (vingt-six pages, contre trois pour l'ambition ou sept pour l'avarice), est tout à fait caractéristique de l'association étroite entre la corruption de l'âme et celle du corps, qui en est le signe exhibé:

$\mathrm{Si}$ un tel remede n'est suffisant, il leur faut mettre en avant les tourmens, les douleurs, les pourritures et corruptions, les puanteurs,

45. Ibid., p. 357.

46. Ibid., p. 362.

47. Ibid., p. 368.

48. Ibid., p. 14. 
les verolles, les chancres qui rongent tout le visage, et autres parties du $\operatorname{corps}^{49}$.

Le discours sur la passion amoureuse est ainsi lui-même le reflet de la certitude plus profonde que l'apparence humaine, lorsqu'elle n'est pas déjà clairement marquée des stigmates de la corruption, n'est jamais que le masque de cette dernière, profondément inscrite dans la chair humaine (chair qui revêt bien volontiers en cette circonstance les apparences de la femme). Ainsi Du Chesne cite-t-il deux exemples de remède particulièrement efficaces. Le premier est celui auquel eut recours Hypatia pour guérir un jeune homme de la passion qu'il avait pour elle:

Elle fit provision de quelques drapeaux sanglans et infectez de ses mauvaises menstrues, et l'ayant fait un jour entrer en sa chambre faignant luy vouloir donner contentement, hausse sa robbe, luy descouvre une ordure si contagieuse, et luy tient telles ou semblables paroles: Mon amy, regarde un peu, je te prie, quelle part tu as laissé ton jugement en sequestre: considere que c'est que tu aimes: examine de pres quel motif t'induit à caresser tant d'immondicité fardée et desguisée d'une vaine beauté.

Le second est imputé à une femme aimée de Raymond Lulle qui, pour le décourager, le fit venir jusqu’à son lit, "descouvrit son tetin gauche et luy fit voir un grand chancre qui l'avoit presque tout rongé, spectacle si hideux, que cela luy servit d'un seur et specifique remede» ${ }^{50}$. Au-delà de leur vertu thérapeutique, on peut s'interroger sur la valeur de ces exemples en termes spirituels ${ }^{51}$ : tous deux (et Du Chesne insiste sur le rôle frappant de l'image) relèvent d'une pratique exhibitionnisme qui met à nu l'intérieur du corps, corps purulent, coulant et transpirant, à travers ces deux miroirs de l'intériorité que sont le linge souillé et le chancre suintant, passage sordide entre l'intérieur et l'extérieur.

Ce bref tour d'horizon révèle à quel point la définition de la perspective hygiénique et thérapeutique dans laquelle se place le médecin est encore, en ce début de XVII ${ }^{e}$ siècle, étroitement conditionnée par

49. Ibid., p. 60.

50. Ibid., p. 61 sq.

5I. Voir l'usage que François de Rosset fait de la composante abjecte du corps féminin dans un passage célèbre des Histoires tragiques (p. 235). 
la notion de corruption. Mais la perspective théologique et ontologique venue de l'aristotélisme chrétien est rendue plus aiguë par la crise des Réformes, protestante puis catholique; elle s'incarne et se développe en des traductions sensibles multiples, elles-mêmes toujours restituées en termes polysémiques, qui témoignent d'une véritable obsession. L'angoisse de l'ordure, du chancre, de l'odeur méphitique, s'accentue, pour des raisons plus morales et théologiques que véritablement hygiéniques. Ce faisant, le texte de Du Chesne est lui-même symptomatique d'une approche de l'air qui devient, en raison même de ses liens avec la corruption, purement morbide et signale qu'est déjà présent ce qu'Alain Corbin a magistralement observé pour le siècle suivant:

Par un détour qui nous semble curieux, c'est bien l'angoisse de la mort, de la désunion des parties du corps vivant qui crée ici la fascination. L'air n'est plus tant étudié comme le lieu de la génération ou de l'épanouissement de la vitalité que comme le laboratoire de la décomposition. Observer avec une attention morbide la marche de la dissolution de la substance organique, repérer l'échappement du "ciment» des corps, de cet "air fixé» promu au rang de principal acteur du drame qui se joue, sentir - au sens propre - se défaire la cohésion des mixtes exerce une inquiétante fascination. Il s'agit de suivre le cheminement de la mort dans la matière vivante ${ }^{52}$.

Violaine Giacomotto-Charra

Université de Bordeaux-III

52. A. Corbin, Le miasme et la jonquille, p. 17. 


\section{BIBLIOGRAPHIE}

\section{Textes}

Du Chesne, Joseph, La Morocosmie: ou De la folie, vanité, et inconstance $d u$ Mond, [Lyon, J. de Tournes, 1583], éd. par Lucile Gibert, Genève, Droz, 2009.

—, Le Pourtraict de la santé [1606], Paris, Claude Morel, 1627.

Dupleix, Scipion, La Physique ou sciences des choses naturelles [1603], éd. par Roger Aview, Paris, Fayard, 1990.

—, La Curiosité naturelle, Rouen, Nicolas Angot, 1615.

Quillian, Michel, La Derniere Semaine ou consommation du monde, F. Huby, Paris, 1596.

Rosset, François de, Histoires tragiques, éd. par Anne de Vaucher Gravili, Paris, LP, 1994.

Zabarella, Giacomo, De Rebus Naturalibus libri XXX, Venise, 1590.

-, Commentarii Collegii Conimbricensis S. I. in duos libros de generatione et corruptione Aristotelis Stagirite, Coimbra, 1597.

\section{Travaux}

Corbin, Alain, Le miasme et la jonquille, Paris, Flammarion, 1986.

Deluz, Christiane, "La sphère de l'air troublé», in Observer, lire, écrire le ciel au Moyen Age, éd. par Bernard Ribémont, Paris, Klincksieck, 1991, p. 87-98.

Hiraï, Hiro, "Paracelsisme, néoplatonisme et médecine hermétique dans la théorie de la matière de Joseph Du Chesne à travers son Ad veritatem hermetica medicina (1604)", Archives internationales d'histoire des sciences, 51 (2001), p. 9-37. 
Victoria, Thierry, Un livre de feu dans un siècle de fer. Les lectures de l'Apocalypse dans la littérature française de la Renaissance, Louvain, Peeters, 2009. 